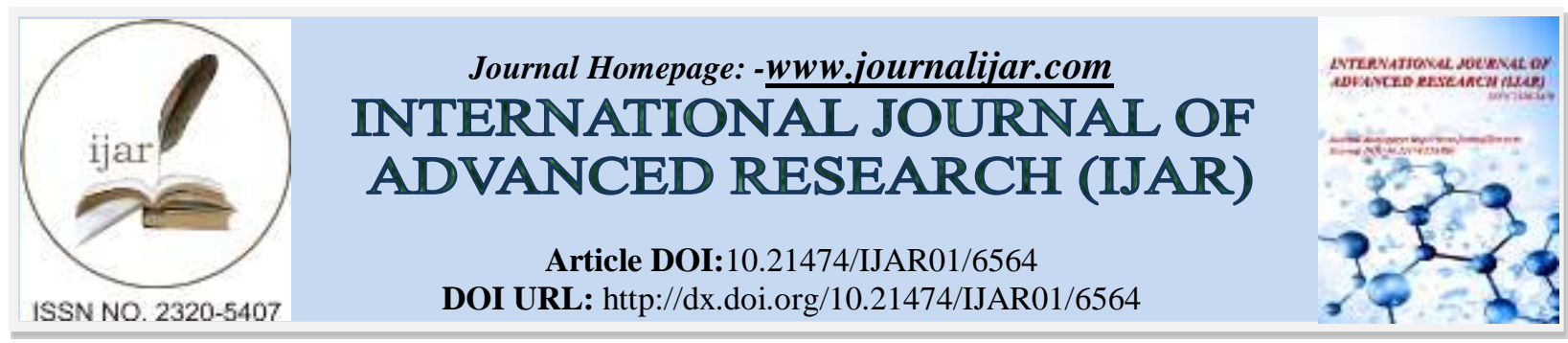

RESEARCH ARTICLE

\title{
THE MAIN REASON OF DENTAL EXTRACTION AMONG YOUNG ADULTS PATIENTS AT UQUDENT IN MAKKAH-SAUDI ARABIA.
}

\author{
Alaa A. Bajafar ${ }^{1}$ and Mazen Al Masri ${ }^{2}$. \\ 1. Resident of Oral And Maxillofacial Surgery at King Fahad General Hospital, Jeddah, Saudi Arabia \\ 2. Assistant professor/consultant of Oral And Maxillofacial Surgery and Implantology at King Abdulaziz \\ University, Jeddah, Saudi Arabia.
}

\section{Manuscript Info}

Manuscript History

Received: 17 December 2017

Final Accepted: 19 January 2018

Published: February 2018

Keywords:-

Sponge, Levantine, Mediterranean, Antibacterial Agent, Pathogenic

Bacteria.

\section{Abstract}

Background: Dental extraction is removal of a tooth from its dental socket in the alveolar bone. Young adults are the population with age ranging from (20-39) years old that they tended to have a good general and periodontal health. Dental extraction is the worst consequence for a diseased tooth so prevention is the best option, So that in order to raise the level of prevention we have to know what is the main reason of dental extraction for this age group .

Materials and Methods: This study is a cross sectional study with retrospective data collection. The study conducted at UQUdent using the R4 system aiming to reach the previous extracted teeth and observe the current extraction cases in order to determine the most common reason of dental extraction among young adults patients at UQUdent.

Results: We found that $53.21 \%$ of the total cases were extracted because it was carious until it reached the level of remaining root stumps . while $37.61 \%$ of the cases were non restorable teeth. The orthodontic reasons and impaction had less effect on the reason of dental extraction with a prevalence of $6.42 \%$ and $2.75 \%$, respectively Conclusion: It can be concluded from this study that the remaining root stumps had occupied the majority of the cases which is the end result of dental decay, while the other reasons had a less effect on the dental extraction.

Copy Right, IJAR, 2018,. All rights reserved.

\section{Introduction:-}

A dental extraction (also referred to as tooth extraction or exodontia) is the removal of a tooth from its dental socket in the alveolar bone. In the past people used to extract their teeth wither if there was a cause or not. After the dentistry evolution, people and most general practitioners was tending to go further toward the conservation if there was any hope from the tooth. Dental extraction is the most surgical procedure that is performed by undergraduate dental student and most general clinicians. Dental extraction is the worst consequence for a diseased tooth despite that there is no other way to treat a hapless tooth except by extracting it and replace it by other means .

There are many reasons that tend the dentist to extract a tooth : 
Dental caries and infection : there are several ways to try and save the tooth. The damaged tooth might need a fullcoverage crown, a root canal treatment, or both. But sometimes even these methods are not enough to keep the tooth functioning well and looking good; it might be better to remove the tooth and replace it with a strong and lifelike dental implant. (1)

Impacted Wisdom Teeth - Early removal of impacted wisdom teeth can prevent damage to neighboring healthy teeth, bone, gum tissue, even nerves and blood vessels. If an impacted wisdom tooth is in a bad position, it's best to remove it before its roots are fully formed. (1)

For orthodontic treatment : Teeth are sometimes extracted when there are too many of them for the size of the dental arches (jaws), a situation known as crowding. After an adequate amount of space is opened up through the extraction of one or more teeth, the remaining teeth can be aligned properly. The teeth most frequently removed for orthodontic reasons are the first premolars, which are right next to the eyeteeth (canines). (1)

Severely periodontal affected teeth that lost most of its supporting structure .

Deciduous teeth : If a Deciduous tooth is out of position or not lost in the right sequence, the permanent tooth underneath it might not erupt normally. In this case, removing the Deciduous tooth could prevent a need for orthodontic treatment later on.

Supernumerary teeth that are blocking the emergence of other teeth .

Tooth that had a vertical root fracture .

Tooth in a fracture line .

Head and neck radiation therapy, to treat or manage tumors, may require extraction of teeth, either before or after radiation treatments .

The Process of Extracting a Tooth : (1)

The first step in any extraction is a radiographic (x-ray) examination to assess the position of the tooth roots and the condition of the surrounding bone. This will allow any possible complications to be anticipated. A thorough medical and drug history is taken, to ensure that you are healthy enough to undergo the procedure .

Tooth extraction is usually carried out with local anesthesia, which will numb the teeth to be removed, and the surrounding bone and gingival tissues. Additional sedatives might also be used, including oral sedatives, nitrous oxide (which is inhaled) and/or conscious sedation, which is given intravenously. The latter is usually required for more complicated (or multiple) tooth extractions.

As the tooth is being removed, steps are taken to ensure the bone that surrounds it isn't damaged. Sometimes, in the process of removing a tooth, a small amount of bone-grafting material is placed into the socket to help preserve the bone volume there. This is particularly important when the extraction is going to be followed at some point by the placement of a dental implant, which needs to fuse to existing bone, or orthodontics, which gently moves teeth through bone.

The most common complications that clinicians encounter during dental extractions: (2)

\section{Bleeding:-}

One of the most common complications of all surgeries is post-operative bleeding. Post-operative bleeding from dental extraction is commonly due to venous bleed from nutrient blood vessels in the supporting bone but can also be due to an arterial source. Other causes of post-operative bleeding may include the failure to debride all granulation tissues from the socket .

\section{Surgical Swelling \& Infection:-}

Post-operative soft-tissue swelling can be a normal part of the healing process. The wound heals by the inflammatory process, which has four cardinal signs: tumor (swelling), rubor (redness), dolor (pain), and calor (heat). The initial clot serves as a wound protector as well as scaffolding for the formation of granulation tissues. Granulation tissues are highly vascularized tissue beds that help bring nutrients and fibroblasts to the wound for repair. Due to the increased blood flow, increased hydrostatic pressure, and increased transudate that contains all the immune cell types and chemotactic factors, swelling is ensured. 


\section{Sinus Perforation:-}

The maxillary sinus is a potential source of complication during the extractions of upper molars. The floor of the sinus is usually the closest to the palatal root of the upper first molars. The floor of the sinus may be so close to the roots that part of it can be removed with the tooth during routine extractions. Other times, the sinus can be easily perforated during traumatic retrieval of broken root tips.

\section{Root Tip in Maxillary Sinus:-}

As mentioned above, the floor of the sinus is closely associated with the maxillary molar roots. If a root tip is pushed into the sinus during extractions, place the patient in an upright position to allow gravity to draw the root tip closer to the perforation. Ask the patient to blow the nose with nostrils closed, then watch for the root tip to appear in view near the perforation for suctioning. One can also try antral lavage, in which saline is injected into the sinus in an attempt to flush the root tip out.

\section{Nerve Injury:-}

The inferior alveolar nerve and artery are both contained within the inferior alveolar canal. The course of this canal is such that it usually runs buccal and slightly apical to the roots of the mandibular molars. During extraction of the mandibular molars, due to the proximity of the roots, the nerve can be traumatized.

Studies on reasons for dental extraction are important because dental extraction is an indicator of dental caries and status of oral health of a population[Naz, 2015] .

But young adults patients (20-39 years old ) are supposed to have a very good general health and their periodontal support is good enough so in this study we will focus on the reasons that lead them to extract their teeth in order to prevent this from happening .

\section{Literature review:-}

There is no available data about the main reason of exodontia for young adults patients at Makkah or the western region of Saudi Arabia but in an other study from Pakistan they found that the $42.2 \%$ of all the cases was extracted due to dental while $30.6 \%$ of cases was extracted for a periodontal disease [Naz , 2015]. Also another study was done among dental patients in Bayelsa State, Nigeria found that dental caries and periodontal disease are the most common cause of dental extraction [-Emmanuel,2015]. In another survey that was done at Scotland to identify the reasons of dental extraction of various tooth types concluded that caries and its sequelae remains the principal reason for loss of all tooth types other than lower incisors which are extracted mainly for periodontal reasons [McCaul LK,2001] . Caries was C the main reason for extraction in patients under 50 years, whereas periodontal disease was the commonest reason in the over-50 age group [Hull PS,1997] . A cross sectional study was done in Oman and revealed that exodontia rates were significantly higher in females, persons older than 12 years, and those living in the coastal areas and they found that a gross dental caries with no salvation problem is the main reason for dental extraction ( 51.9\%) [Khandekar R, 2003 ] . A survey was run in Singapore to identify the main reason for dental extraction and they found at the end that the percentage of teeth extracted due to periodontal reasons and caries were about the same, that is $35.8 \%$ and $35.4 \%$, respectively [ Ong G , 1996 ] . caries was the most frequent reason for tooth extraction (55.4\%), followed by periodontal disease (38.0\%) in a study that was done in Japan [Morita M , 1994 ] . Another study was undertaken in September 1990 to investigate the reasons for permanent tooth extractions in Antigua and they found that the principle reason for extractions was caries $(61.6 \%)$ while Periodontitis $(29.9 \%)$ was the next most frequent reason for extractions and it became the predominant indication for tooth loss after 40 years of age [ Vignarajah S,1993 ]. Also a study was done in 1993 for the reason of dental extraction in western states in Germany and they found that Caries was the reason given for $20.7 \%$ of all extractions; periodontal diseases for $27.3 \%$; caries and periodontal reasons for $18.7 \%$; third molars for $14.7 \%$; prosthetic reasons for $11.2 \%$; orthodontic reasons for $4.1 \%$; trauma for $0.4 \%$ and others for $2.9 \%$. While caries is a major reason in all age groups, periodontal diseases and the combination of caries and periodontal reasons are more frequent than all other reasons for the age groups beyond 40 or $45 \mathrm{yr}$, respectively [Reich E,1993] .

\section{Rationale:-}

Limited studies about the main reason of dental extraction in Makkah region and in the Kingdom of Saudi Arabia . Objective:Identify of the main reason of dental extraction among young adults patients at UQUdent in Makkah . 


\section{Methodology:-}

\section{Study population:-}

109 cases were collected from both genders and it carried out on a period of 6 weeks at UQUdent clinics in Makkah city at both male and female departments. The study population was reviewed on the system for the past dental treatment at the clinics and the cases that has been involved were done in the past 6 months. The cases that came to the hospital in the period of our study were included and data was collected from them .

\section{Inclusion criteria:-}

Age group 20-39 years of both genders.

Must had extraction at UQUDent clinics before or during the period of the study .

\section{Exclusion criteria:-}

Age group beyond 20 years or above 39 years of both genders .

\section{Study design:-}

A cross-sectional study with retrospective data collection was conducted to determine the most common reason of dental extraction at UQUdent n Makkah- Saudi Arabia on a period of the last 6 months .

\section{Data Collection:-}

It involved two parts:-

1st part: This study is about reviewing the system for the past dental treatment at UQUdent clinics and the cases that has been involved were in the past 6 months. The data that was collected from the system and entered into the data table that focused mainly on the reason of extraction according to the available data and radiographs also and it was assigned into the four main category which are remaining root stump, non restorable tooth ( a decayed tooth that has a part of its crown but it's indicated for extraction for another reason ), impaction and for orthodontic treatment . Also it involved some data that is related to the extracted tooth like ( the endodontic condition of the tooth, the periodontal condition and the bone loss ) and also some demographic data of the patients which are the age and gender only, and also the general health of the patients was evaluated for any medical condition that compromise the patient and finally the oral hygiene of the patients was measured according to the visible calculus on $\mathrm{x}$-ray and the oral hygiene measures of the patient. And a copy from the X-ray was kept for a documentation purpose .

2nd part: was the cases that came to the hospital in the period of the study for extraction were included and the data was collected in the same manner.

\section{Statistical Analysis:-}

The data were collected and entered using Microsoft Excel program to come up with the clinical correlations and statistical observations and the prevalence was established manually

\section{Ethical considerations:-}

An ethical approval was obtained from the clinic manager Dr.Hasan Hazzazi, umm alQura University, makkah, Saudi Arabia.

All information was kept confidential and was not accessed except for the purpose of the research.

\section{Results:-}

109 cases were collected in this study, 74 cases were females while 35 cases were males. The reason for extraction was determined for each individual case and $37.61 \%$ of cases was non restorable tooth while the prevalence for remaining root stump was $53.21 \%$, also $6.42 \%$ of the cases were extracted for orthodontic reason and $2.75 \%$ of cases were extracted because of impaction. So the more than half of the cases were extracted because it was a remaining root stump.

We also found that $61.64 \%$ of cases were in the age ranged from $20-29$ years while $37.61 \%$ of cases were in the age ranged from $30-39$ years.

Also we found that $76.14 \%$ of the cases had mild bone loss while $23.86 \%$ of the cases had moderate to severe bone loss. 
Table 1:- Prevalence of the main reason of dental extraction $(n=109)$

\begin{tabular}{|l|l|l|}
\hline & $\mathrm{N}$ & $\%$ \\
\hline Remaining root stump & 58 & $37.61 \%$ \\
\hline Non restorable tooth & 41 & $53.21 \%$ \\
\hline Orthodontic reason & 7 & $6.42 \%$ \\
\hline impaction & 3 & $2.75 \%$ \\
\hline
\end{tabular}

Figure 1:- prevalence of the reason of dental extraction

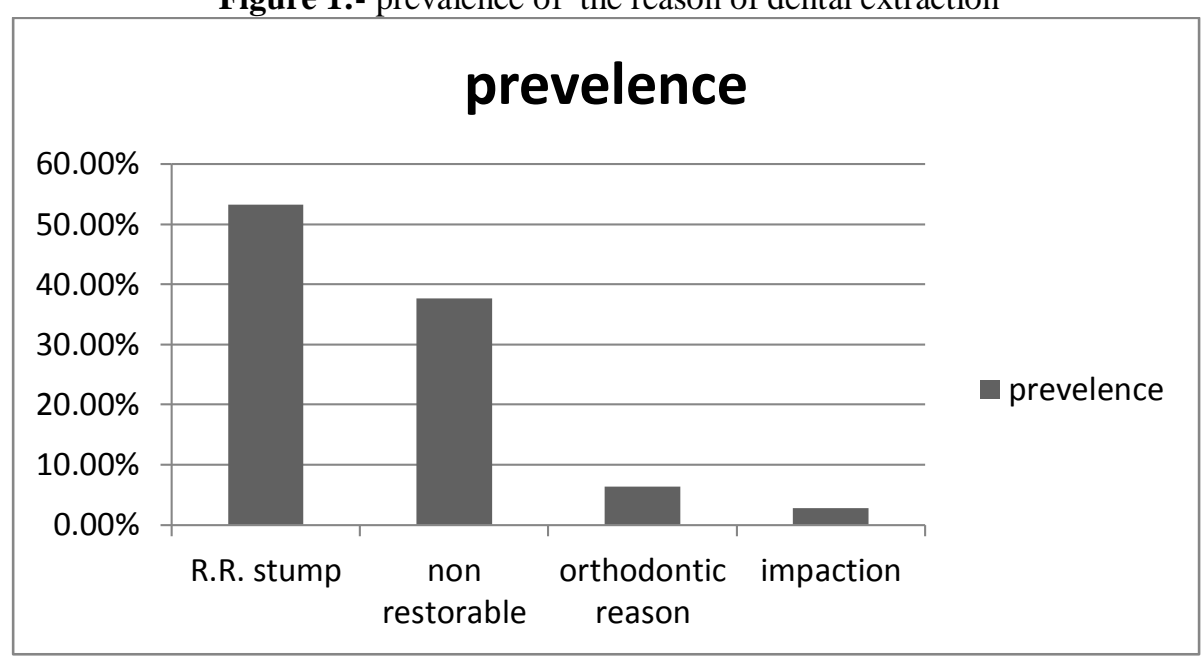

\section{Discussion:-}

This study focused on evaluating the dental concern of the population in Makkah city through determining the main common reason of dental extraction which is the end result of a diseased tooth. As limited information was available at Makkah and the whole kingdom of Saudi Arabia about the reason of exodontia, so that the results of this study will help us to measure the services that is available for the population and the interest of them for seeking the dental care.

As we can see that $37.61 \%$ of the cases was extracted due to non restorable crowns which a consequences of tooth decay, and when the decay develop into more aggressive form it will make a non restorable crowns into remaining root stumps which to be found in $53.21 \%$ in the cases that is the majority. This can give us the indication of a poor dental awareness about dental care as most of the cases dropped into the category of the remaining root stump which have not any treatment other than extraction . while the orthodontic reason and impaction had the least effect on tooth extraction which are contributed to be $6.42 \%$ and $2.75 \%$ respectively.

When the endodontic condition of the remaining root stump was evaluated we found that $5.17 \%$ of the cases had an endodontic treatment, which indicates that the current population didn't give their teeth an appropriate treatment from the beginning. That means the awareness of the population towards the dental health is very low and we have increase it for these population to encounter the huge extraction cases.

Most of the cases had mild bone loss ( $76.14 \%$ ) which means that the extracted teeth has no relation with an aggressive periodontal disease, also it support the idea that a young adults population will have good periodontal support that no obvious relation between the extraction and the periodontal disease .

$61.46 \%$ of the cases was extracted in the age group (20-29) years which means that the tooth life cycle ends earlier in our population and we have to focus on how to increase the tooth life cycle in the population by increasing the seek for dental health and the awareness towards the dental health.

As a very limitation of the study that the R4 system in UQUdent dental clinics don't record the socioeconomic status of the patients which may have a significant impact on the results . 
Our recommendation and the next step for this study is to identify the causes that accelerates to tooth lifecycle at our community and how can we prevent that from happening and try retain a normal carious tooth as long as we can

\section{Conclusion:-}

It can be concluded from this study that the a remaining root stumps had occupied the majority of the cases which is the end result of dental decay . while the other reasons had a less effect on dental extraction. And when the endodontic condition of the tooth was evaluated we found that only few cases went through an RCT which means that the population needs more education about the dental health .

The dental awareness of the current population towards the dental health is very low and we have to increase it .

Also majority of the cases were in a younger cases which means an acceleration in tooth lifecycle in our community

Finally, as our population tends to a good general health and good periodontal health also, we figured out that the dental extraction in this age group had no relation with a severe bone loss so that the periodontal disease is not regular causes for dental extraction at this age group .

\section{References:-}

1. http://www.goldcanyoncontemporarydentistry.com/library/7787/Extractions.html

2. Angelillo IF , Nobile CG, Pavia M : Survey of reasons for extraction of permanent teeth in Italy, Community Dent Oral Epidemiol. , 24(5), 336-40, 1996.

3. Emmanuel E. , Dotimi D. , Idumesaro N. , Usiholo J., Apiakise E. , Gambia G. and Ayilbatonye N. : An assessment of prevalent dental ailments, treatments and reasons for exodontia among dental patients in Bayelsa State, Nigeria, PJI , 1(2), 024-030, 2015.

4. Hull PS , Worthington HV, Clerehugh V, Tsirba R, Davies RM, Clarkson JE : The reasons for tooth extractions in adults and their validation,J Dent ,25(3-4) , 233-7, 1997.

5. Khandekar R, Fouad Z, Al-Khusaibi AM : Magnitude and determinants of exodontia in South Batinah region of Oman: A cross sectional study, J Sci Res Med Sci. , 5(1-2) , 21-5 , 2003.

6. McCaul LK , Jenkins WM , Kay EJ : The reasons for the extraction of various tooth types in Scotland: a 15year follow up , J Dent , 29(6), 401-7, 2001.

7. Morita M, Kimura T, Kanegae M, Ishikawa A, Watanabe $\mathrm{T}:$ Reasons for extraction of permanent teeth in Japan , Community Dent Oral Epidemiol , 22(5 Pt 1):303-6, 1994.

8. Naz F. : reasons for extraction in permenant dentition a study in tertiary case setting in pakistan, JPDA, 2015

9. Ong G, Yeo JF, Bhole S : A survey of reasons for extraction of permanent teeth in Singapore, Community Dent Oral Epidemiol , 24(2):124-7, 1996.

10. Reich E, Hiller KA : Reasons for tooth extraction in the western states of Germany, Community Dent Oral Epidemiol , 21(6):379-83, 1993 .

11. Vignarajah S : Various reasons for permanent tooth extractions in a Caribbean population-Antigua, Int Dent $\mathbf{J}$ , 43(3):207-12, 1993. 